\title{
Experimental overview of correlations in small collision systems
}

\section{A brief history}

\author{
Soumya Mohapatra ${ }^{1, \star}$ \\ ${ }^{1}$ Department of Physics, Columbia University
}

\begin{abstract}
The azimuthal anisotropies of particle yields observed in relativistic heavy ion collisions have been traditionally considered as a strong evidence of the formation of a deconfined quark-gluon plasma in these collisions. However multiple recent measurements in $p p$ and $p / \mathrm{D} / \mathrm{He}+\mathrm{A}$ collisions show similar features as those observed in heavy ion collisions, indicating the possibility of the production of such a deconfined medium in smaller collision systems. This paper presents a comprehensive summary of such measurements in small systems. It includes measurements of identified and inclusive two-particle correlations in $\Delta \phi$ and $\Delta \eta$, with different procedures used to subtract the dijet contributions, as well as measurements of multi-particle cumulants $c_{n}\{2-8\}$. The traditional cumulant measurements confirm presence of collective phenomena in $p+\mathrm{A}$ collisions, but are biased by non-flow correlations and are not able to provide evidence for collectivity in $p p$ collisions. To address this, a new subevent cumulant method that further suppresses the contribution non-flow effects was developed, whose measurements are also discussed.
\end{abstract}

\section{Introduction}

In relativistic heavy ion collisions, such as those at the RHIC and at the LHC, the colliding nuclei are heated to high enough temperatures such that the quarks and gluons that are ordinarily confined within nucleons get de-localized and a new state of matter called the quark-gluon plasma (QGP) is created. The medium expands anisotropically driven the pressure gradients between the medium and the outside vacuum, cooling down as it expands and finally hadronizes. The final particle yields in the azimuthal $(\phi)$ direction are typically parameterized as a Fourier series [1]:

$$
d N / d \phi \propto\left(1+2 \sum v_{n} \cos n\left(\phi-\Psi_{n}\right)\right.
$$

where the $v_{n}$ and $\Phi_{n}$ denote the magnitude and orientation of the single-particle anisotropies. Due to their hydrodynamic origin in heavy ion collisions, the $v_{n}$ are often called flow harmonics.

Such azimuthal anisotropies were not expected to arise in proton-proton $(p p)$ and proton-nucleus $(p+\mathrm{A})$ collisions where the QGP was not expected to be formed. However in 2010 the CMS collaboration showed that in high-multiplicity $p p$ collisions, two-particle correlations (2PCs) in relative azimuthal angle $\left(\Delta \phi=\phi^{a}-\phi^{b}\right)$ and pseudorapidity separation $\left(\Delta \eta=\eta^{a}-\eta^{b}\right)$, where the superscripts $a$ and $b$ label the two particles used in the correlation, showed the presence of a long range correlation structure along $\Delta \eta$ at $\Delta \phi \sim 0$ [2]. This structure, commonly called "ridge", is also seen in heavy ion

^e-mail: soumya@cern.ch 

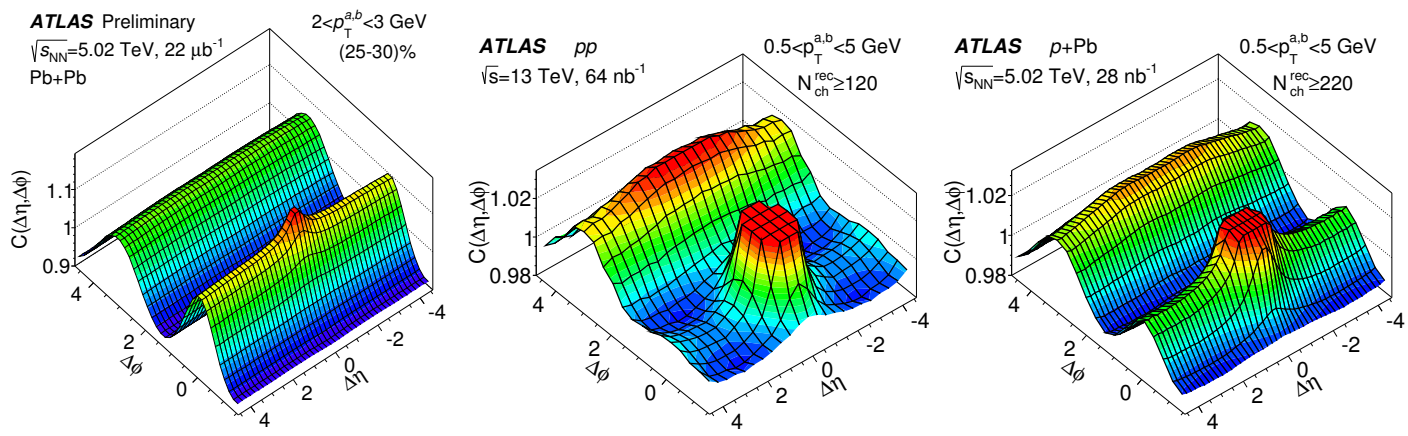

Figure 1. Two particle correlations $(C(\Delta \eta, \Delta \phi))$ in $\mathrm{Pb}+\mathrm{Pb}($ left), $p p$ (center) and $p+\mathrm{Pb}$ (right) collisions. The $\mathrm{Pb}+\mathrm{Pb}$ results are for $2<p_{\mathrm{T}}^{\mathrm{a}, \mathrm{b}}<3 \mathrm{GeV}$ and for the $25-30 \%$ centrality interval. The $p p$ results are for $0.5<p_{\mathrm{T}}^{\mathrm{a}, \mathrm{b}}<$ $5 \mathrm{GeV}$ and for events having more than 120 reconstructed charged particles with $p_{\mathrm{T}}>0.4 \mathrm{GeV}$. The $p+\mathrm{Pb}$ results are for $0.5<p_{\mathrm{T}}^{\mathrm{a}, \mathrm{b}}<5 \mathrm{GeV}$ and for events having more than 220 reconstructed charged particles with $p_{\mathrm{T}}>0.4 \mathrm{GeV}$. The plots are from Refs. [4, 5]

collisions and arises there due to collective flow. Thus its presence in $p p$ collisions indicated the possibility of collective effects being present in $p p$ collisions. The left and middle panels of Figure 1 compare the ridge seen in $\mathrm{Pb}+\mathrm{Pb}$ and $p p$ collisions, respectively. The $p p$ ridge is seen to be considerably weaker that the $\mathrm{Pb}+\mathrm{Pb}$ ridge. The weakness of the ridge in $p p$ collisions made it impossible to use the techniques that were developed for and used in analysis of $2 \mathrm{PCs}$ in $\mathrm{A}+\mathrm{A}$ collisions. Further more, the $p p$-ridge could be explained by initial state models based on gluon saturation [3], and this was accepted by the heavy ion community as its origin.

\section{Collectivity in $p+\mathbf{A}$ collisions}

In 2012 the analysis of the first $p+\mathrm{Pb}$ collision data at the LHC from ATLAS, ALICE and CMS collaborations showed the presence of the ridge in $p+\mathrm{Pb}$ collisions as well [6-10]. Unlike the $p p$ case, the long-range correlations in $p+\mathrm{Pb}$ were strong enough (see Figure 1 right panel), and the multiplicities were large enough, that the techniques used for analysis of $2 \mathrm{PCs}$ in $\mathrm{A}+\mathrm{A}$ collisions could be applied. The $2 \mathrm{PC}$ analysis indicated that the long-range correlations were indeed arising from single-particle anisotropies, $v_{n}$, which could be extracted using the 2PCs [7]. The $p+\mathrm{Pb}-v_{n}$ were found to be remarkably similar to than seen in A+A collisions. In fact, after some trivial scalings, the $v_{2}-v_{4}$ in $p+\mathrm{Pb}$ had exactly the same $p_{\mathrm{T}}$ dependence as the $v_{n}$ in $\mathrm{Pb}+\mathrm{Pb}$ collisions [9]. This is demonstrated in Figure 2. The left panels show the comparison of $v_{2}-v_{4}$ as a function of $p_{\mathrm{T}}$ in $\mathrm{Pb}+\mathrm{Pb}$ and $p+\mathrm{Pb}$ collisions. The centrality interval of $55-60 \%$ in the $\mathrm{Pb}+\mathrm{Pb}$ data is chosen such that it approximately corresponds to the multiplicity range of the $p+\mathrm{Pb}$ data to which it is compared $\left(220 \leq N_{\mathrm{ch}}^{\mathrm{rec}}<260\right)$. The right panels of Figure 2, the $\mathrm{Pb}+\mathrm{Pb}$ data are rescaled horizontally by a constant factor of 1.25 , and the $v_{2}$ and $v_{4}$ are also down-scaled by an empirical factor of 0.66 to match the $p+\mathrm{Pb}$ data. The factor if 1.25 is to account for the difference in the $\left\langle p_{\mathrm{T}}\right\rangle$ between the $\mathrm{Pb}+\mathrm{Pb}$ and $p+\mathrm{Pb}$ data [11], while the empirical factor of 0.66 accounts for the differences in the collision geometry in the two systems.

Additionally, measurement of $v_{n}$ via multi-particle cumulants and Lee-Yang Zeroes (LYZ) methods in $p+\mathrm{Pb}$ collisions also exhibited similarities with $\mathrm{Pb}+\mathrm{Pb}$ collisions [10]. Figure 3 shows the $v_{2}$ obtained from 4, 6 and 8-particle cumulants $\left(v_{2}\{4\}, v_{2}\{6\}\right.$ together with the $v_{2}$ from 2PCs $\left(v_{2}\{2, \Delta \eta>2\}\right)$ 

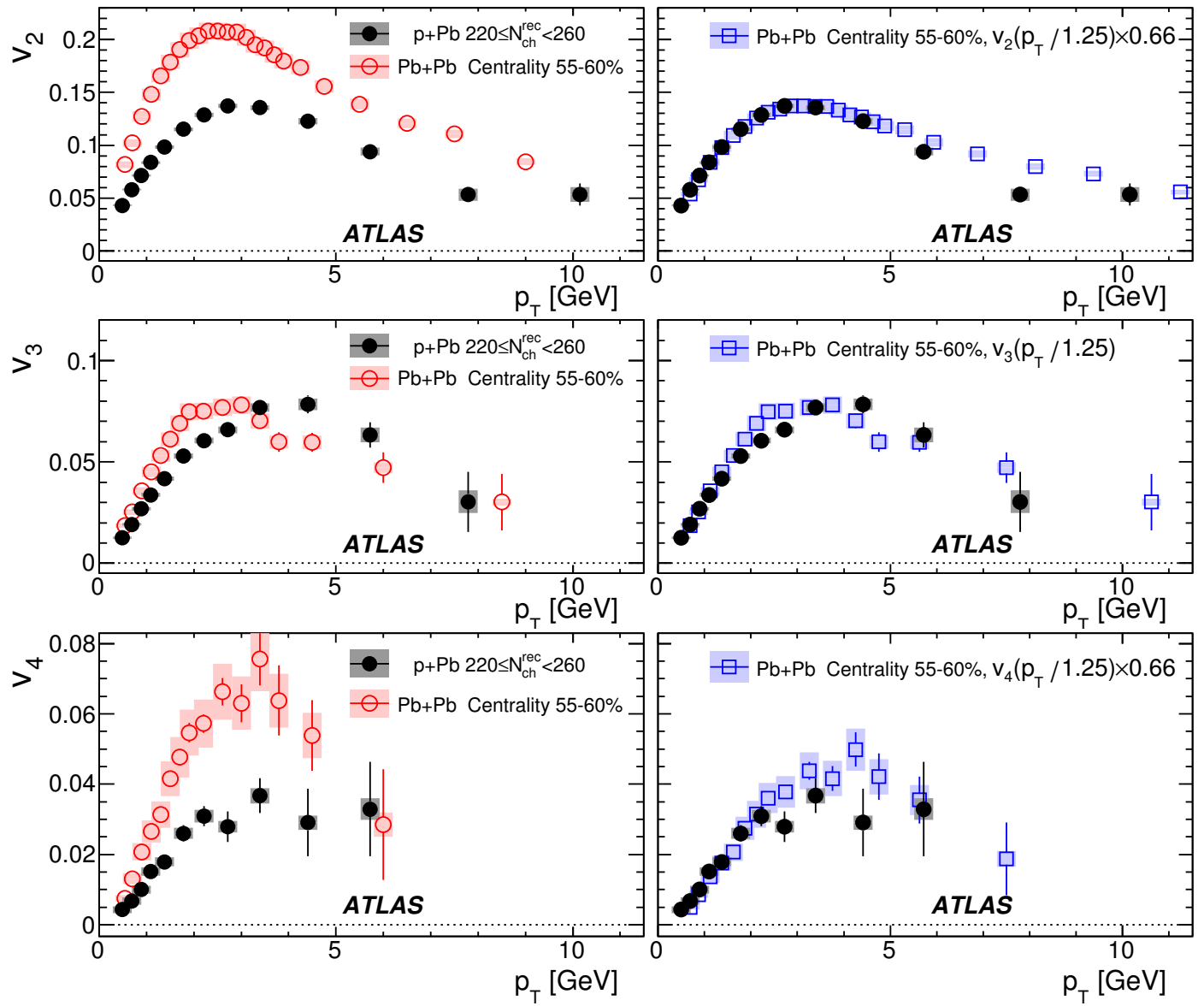

Figure 2. The coefficients $v_{2}$ (top row), $v_{3}$ (middle row) and $v_{4}$ (bottom row) as a function of $p_{\mathrm{T}}$ compared between $p+\mathrm{Pb}$ collisions with $220 \leq N_{\mathrm{ch}}^{\text {rec }}<260$ and $\mathrm{Pb}+\mathrm{Pb}$ collisions in 55-60\% centrality. The left column shows the original data with their statistical (error bars) and systematic uncertainties (shaded boxes). In the right column, the same $\mathrm{Pb}+\mathrm{Pb}$ data are rescaled horizontally by a constant factor of 1.25 , and the $v_{2}$ and $v_{4}$ are also down-scaled by an empirical factor of 0.66 to match the $p+\mathrm{Pb}$ data. The Figures are taken from Ref. [9].

in $\mathrm{Pb}+\mathrm{Pb}$ (left) and $p+\mathrm{Pb}$ collisions (right). It is seen that in both systems, the $v_{2}$ obtained from the 4 , 6 and 8 particle cumulants are identical within systematic uncertainties, and smaller than the $2 \mathrm{PC}-\mathrm{v}_{2}$. This ordering of the $v_{2}$ measured via these different methods is considered a characteristic of flow phenomena [10].

Further more, $v_{n}$ measurements in other small-large collision systems, namely $d+\mathrm{A}$ and ${ }^{3} \mathrm{He}+\mathrm{A}$ collisions, also demonstrated similarities with A+A collisions, and were well reproduced by hydrodynamic calculations [12-16]. A summary of such results from the PHENIX collaboration is shown in Figure 4. These and other measurements established with considerable certainty the presence of collective behavior in $p / \mathrm{D} / \mathrm{He}+\mathrm{A}$ collisions. 


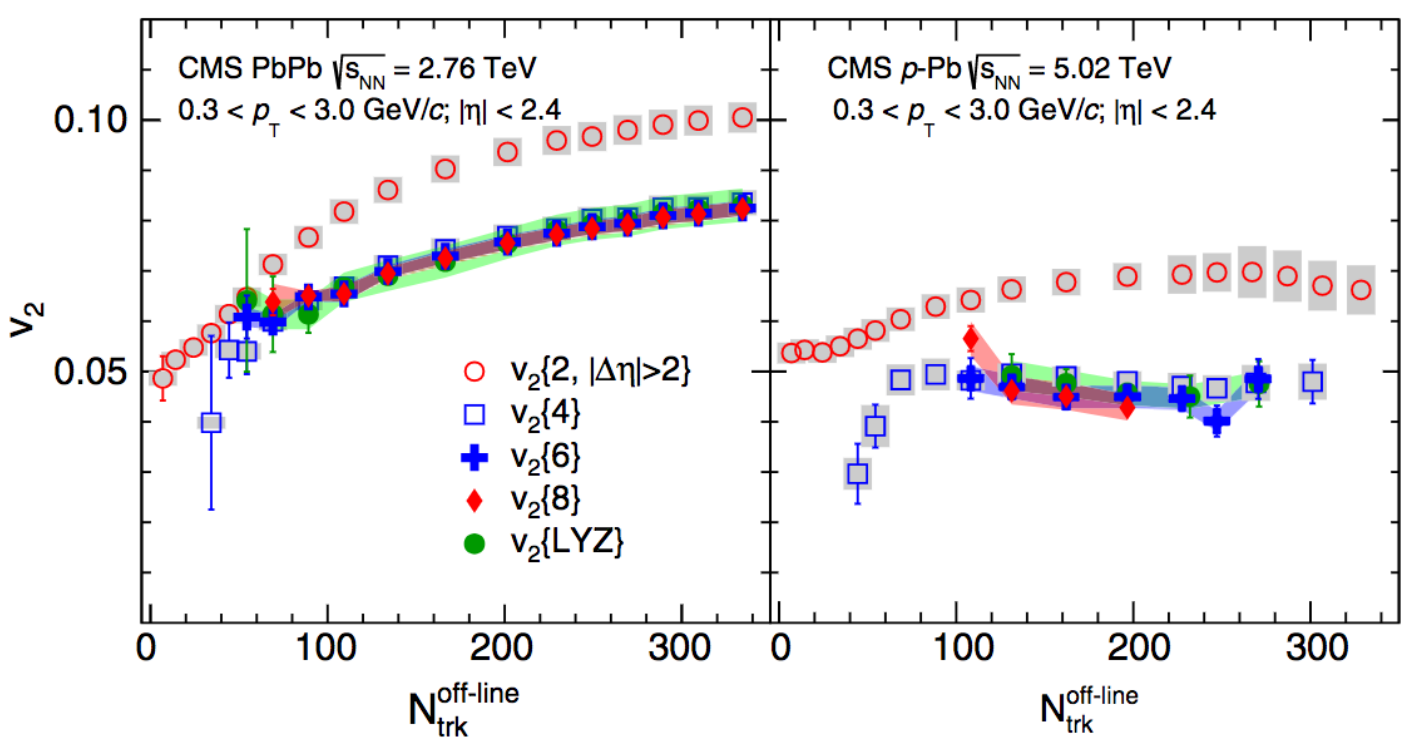

Figure 3. The multiplicity dependence of the $v_{2}$ obtained from 2PCs $\left(v_{2}\{2, \Delta \eta>2\}\right)$, from 4, 6 and 8-particle cumulants $\left(v_{2}\{4\}, v_{2}\{6\}\right.$ and $\left.v_{2}\{8\}\right)$, and from Lee-Yang Zeroes (LYZ) methods. Results are for particles with $0.3<p_{\mathrm{T}}<3 \mathrm{GeV}$. The left panel corresponds to $\mathrm{Pb}+\mathrm{Pb}$ collisions with $\sqrt{s_{\mathrm{NN}}}=2.76 \mathrm{TeV}$. The right panel corresponds to $p+\mathrm{Pb}$ collisions with $\sqrt{s_{\mathrm{NN}}}=5.02 \mathrm{TeV}$. The error bars and bands indicate statistical and systematic uncertainties, respectively. Figure taken from Ref. [10].

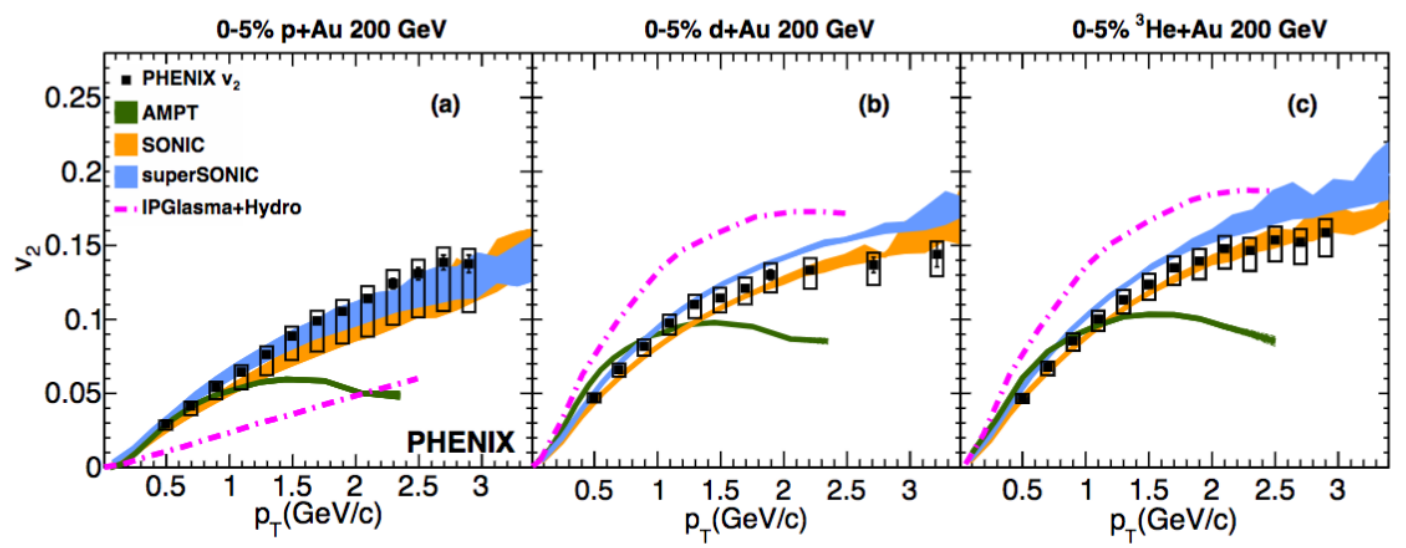

Figure 4. The $p_{\mathrm{T}}$ dependence of the $v_{2}$ measured in $0-5 \%$ most central $p+\mathrm{Au}$ (left), $\mathrm{D}+\mathrm{Au}$ (center) and ${ }^{3} \mathrm{He}+\mathrm{Au}$ collisions at $\sqrt{s_{\mathrm{NN}}}=200 \mathrm{GeV}$. Also shown on the plots are theoretical calculations from the AMPT, SONIC, superSONIC and IPGlasma+Hydro. Figure taken from [16]. 


\section{Long-range correlations in $p p$ collisions}

These measurements thus necessitated revisiting the $p p$ long-range correlations to perform a more detailed analysis along the lines of the $p+\mathrm{Pb}$ measurements and extract the $v_{n}$ harmonics. However unlike the $p+\mathrm{Pb}$ measurements, the long-range correlation in $p p$ collisions are much weaker compared to the other features seen in the $2 \mathrm{PC}$, namely, the near-side jet peak at $\Delta \eta, \Delta \phi \sim(0,0)$, and the away-side jet correlation at $\Delta \phi \sim \pi$. The near-side peak can be removed by focussing on the 1D correlation, $C(\Delta \phi)$, obtained by by projecting the long-range $(|\Delta \eta|>2)$ part of the correlation on to the $\Delta \phi$-axis. This is shown in the left panel of Figure 5 for $p p$ events at $\sqrt{s}=13 \mathrm{TeV}$, that have more that 120 reconstructed charged particle tracks with $p_{\mathrm{T}}>0.4 \mathrm{GeV}\left(N_{\mathrm{ch}}^{\mathrm{rec}}\right)$. While the $\Delta \eta$ cut removes the near-side peak, the away-side jet peak still remains and dominates the $2 \mathrm{PC}$, and must be somehow removed, prior to extracting the $v_{n}$ from the correlation functions. This was recently done by the ATLAS collaboration who performed a "Template-fit" procedure to remove the contribution of the away-side jet to the 1D 2PC [5, 17]. In this procedure, the measured $C(\Delta \phi)$ distributions are assumed to result from a superposition of a "peripheral" $C(\Delta \phi)$ distribution, $C^{\text {periph }}(\Delta \phi)$, scaled up by a multiplicative factor and a constant modulated by $\cos (n \Delta \phi)$ for $n \geq 2$. The resulting template fit function,

$$
C^{\text {templ }}(\Delta \phi)=C^{\text {ridge }}(\Delta \phi)+F C^{\text {periph }}(\Delta \phi)
$$

where

$$
C^{\mathrm{ridge}}(\Delta \phi)=G\left(1+\sum_{n=2}^{\infty} 2 v_{n, n} \cos (n \Delta \phi)\right),
$$

has free parameters $F$ and $v_{n, n}$. The parameter $F$ is the multiplicative factor by which the $C^{\text {periph }}(\Delta \phi)$ is scaled. The coefficient $G$, which represents the magnitude of the combinatoric component of $C^{\text {ridge }}(\Delta \phi)$, is fixed by requiring that the integral of $C^{\text {templ }}(\Delta \phi)$ be equal to the integral of the measured $C(\Delta \phi): \int_{0}^{\pi} d \Delta \phi C^{\mathrm{templ}}(\Delta \phi)=\int_{0}^{\pi} d \Delta \phi C(\Delta \phi)$. In the ATLAS analysis, the $0 \leq N_{\mathrm{ch}}^{\mathrm{rec}}<20$ multiplicity interval is used to produce $C^{\text {periph }}(\Delta \phi)$. The right panel of Figure 5 shows an example of the template fitting for the $N_{\text {ch }}^{\text {rec }}>120$ multiplicity interval. It is seen that the template describes the data quite well, reproducing both the near-side ridge as well as the narrowing of the away-side peak from low- $N_{\mathrm{ch}}^{\mathrm{rec}}$ to high- $N_{\mathrm{ch}}^{\mathrm{rec}}$ events.

Once the long-range correlation is extracted from the template fits, the single-particle anisotropies $v_{n}$ can be extracted, following the standard procedure used in heavy ion analyses [1], where the Fourier coefficients $v_{n, n}$ of the $2 \mathrm{PC}$ (Eq. (3)) are related to the $v_{n}$ as:

$$
v_{n, n}\left(p_{\mathrm{T}}^{\mathrm{a}}, p_{\mathrm{T}}^{\mathrm{b}}\right)=v_{n}\left(p_{\mathrm{T}}^{\mathrm{a}}, p_{\mathrm{T}}^{\mathrm{b}}\right)
$$

The left panel of Figure 6 shows the $v_{2}$ in $13 \mathrm{TeV}$ and $5.02 \mathrm{TeV} p p$ collisions obtained from the template fits. Also shown for comparison are the $v_{2}$ values in $p+\mathrm{Pb}$ collisions at $\sqrt{s_{\mathrm{NN}}}=5.02 \mathrm{TeV}$. Very interestingly it is observed that the $p p v_{2}$ is independent of $N_{\mathrm{ch}}^{\mathrm{rec}}$, implying that the long range correlation is not a feature that is unique to high multiplicity events only, but in fact is present at all multiplicities. The $p+\mathrm{Pb} v_{2}$ does show a dependence on $N_{\mathrm{ch}}^{\mathrm{rec}}$ and in always larger than the $p p v_{2}$, possibly arising from geometrical effects that re present in $p+\mathrm{Pb}$ but not in $p p$ collisions. However, at the lowest multiplicities the $p+\mathrm{Pb} v_{2}$ values seem to approach the $p p v_{2}$ values. Another feature that is observed is that the $p p v_{2}$ values at 5.02 and $13 \mathrm{TeV}$ are consistent with each other, implying a collision energy independence of the $p p v_{2}$ similar to what in observed in $\mathrm{A}+\mathrm{A}$ collisions $[4,18]$. The right panel of Figure 6 shows the $v_{2}$ as a function of $p_{\mathrm{T}}$, for the same three datasets. As seen in the $N_{\text {ch }}^{\text {rec }}$ dependence, the $v_{2}$ values are consistent between the 5.02 and $13 \mathrm{TeV} p p$ within the systematic uncertainties, over the measured $p_{\mathrm{T}}$ range. The $p p v_{2}$ values are smaller than the $p+\mathrm{Pb} v_{2}$ across 

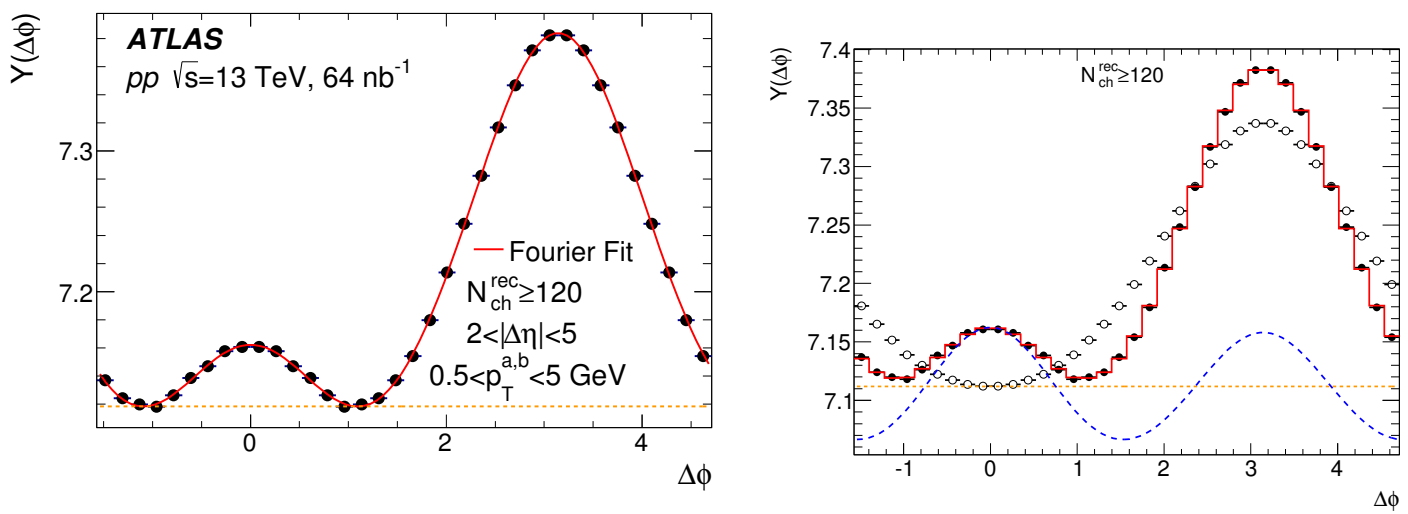

Figure 5. Left panel : The 1D two-particle correlation, in $p p$ events at $\sqrt{s}=13 \mathrm{TeV}$. The $1 \mathrm{D}$ correlations are obtained by projecting the long range $(\Delta \eta>2)$ part of the $2 \mathrm{PC}$ to $\Delta \phi$. The plot is for events with more that 120 reconstructed tracks, and for $0.5<p_{\mathrm{T}}^{\mathrm{a}}, p_{\mathrm{T}}^{\mathrm{b}}<5.0 \mathrm{GeV}$. The continuous line is drawn only to guide the eye. Right panel: Template fit to the 1D 2PC. The template fitting includes second-order, third-order and fourth-order harmonics. The solid points indicate the measured $2 \mathrm{PC}$, the open points and curves show different components of the template (see legend). The Figures are taken from Ref. [5].
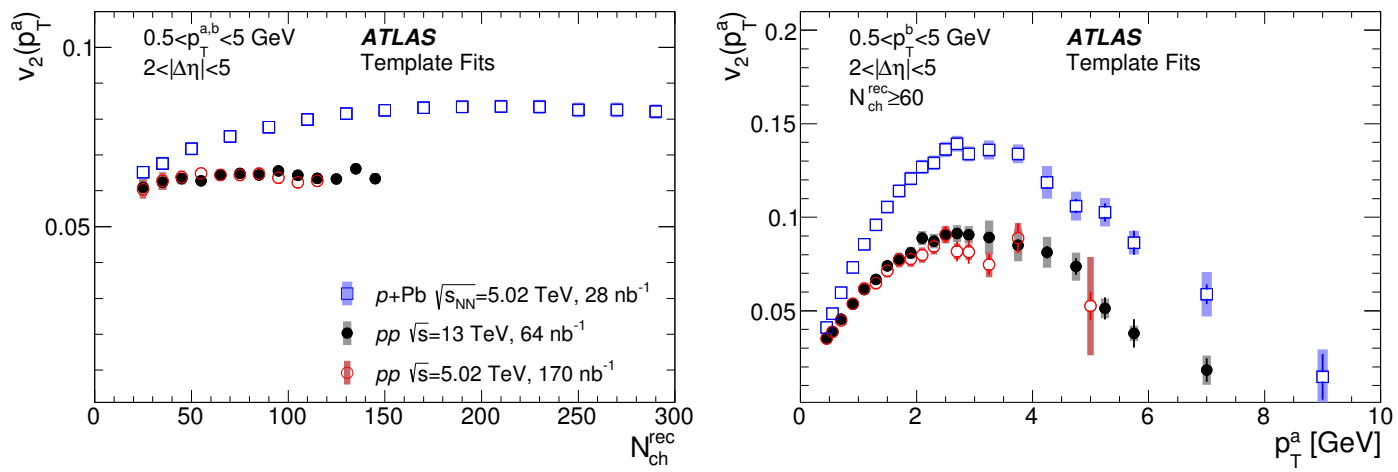

Figure 6. Left panels: comparison of the $v_{2}$ obtained from the template fitting procedure in the $13 \mathrm{TeV} p p$, $5.02 \mathrm{TeV} p p$, and $5.02 \mathrm{TeV} p+\mathrm{Pb}$ data, as a function of $N_{\mathrm{ch}}^{\mathrm{rec}}$. The results are for $0.5<p_{\mathrm{T}}^{\mathrm{a}}, p_{\mathrm{T}}^{\mathrm{b}}<5 \mathrm{GeV}$. Right panels: the $p_{\mathrm{T}}$ dependence of the $v_{2}$ for the $N_{\mathrm{ch}}^{\text {rec }} \geq 60$ multiplicity range. The error bars and shaded bands indicate statistical and systematic uncertainties, respectively. The Figures are taken from Ref. [5].

the whole $p_{\mathrm{T}}$ range. However the shapes of the $v_{2}\left(p_{\mathrm{T}}\right)$ are quite similar between the $p p$ and $p+\mathrm{Pb}$ collisions. This is seen in Figure 7 where the $p p v_{2}$ is scaled by an empirical factor of 1.51 , choosen such that the maxima of the two $v_{2}\left(p_{\mathrm{T}}\right)$ match. This similarity between the the $v_{2}$ in these two systems, and by extension to the $v_{2}$ in $\mathrm{A}+\mathrm{A}$ (see Figure 2) again suggests similar origin for the $v_{n}$ in the three systems. 


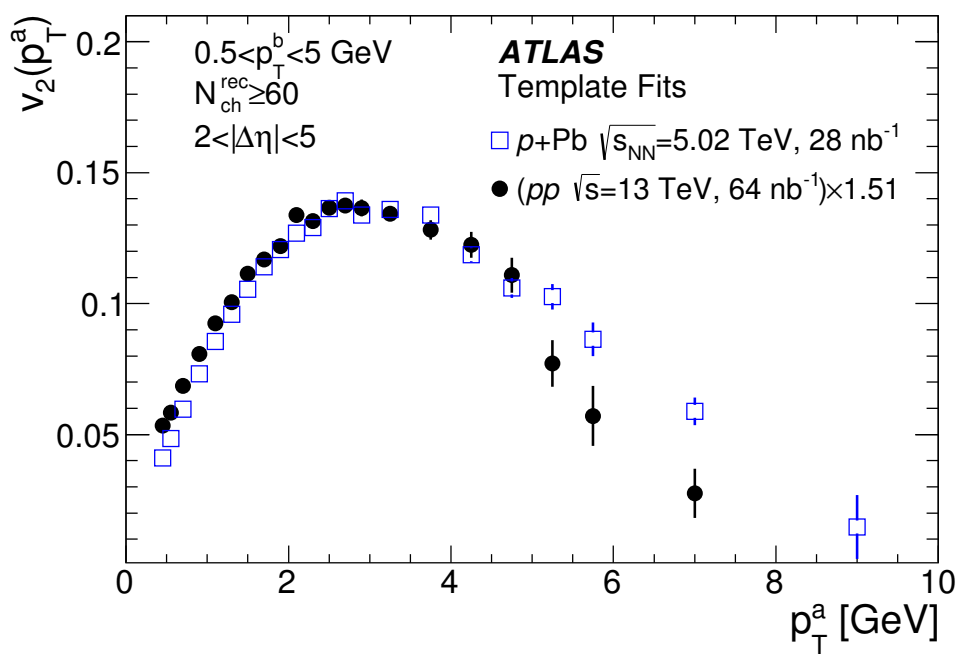

Figure 7. Comparison of the shapes of the $v_{2}\left(p_{\mathrm{T}}\right)$ in $13 \mathrm{TeV} p p$ and $5.02 \mathrm{TeV} p+\mathrm{Pb}$ data. The $p p v_{2}$ has been scaled by a factor of 1.51 along the $y$-axis in order to match the maximum of the $v_{2}$ in the two data sets. The results are for $N_{\mathrm{ch}}^{\mathrm{rec}} \geq 60$. The error bars indicate statistical uncertainties. The Figures are taken from Ref. [5].

Besides two-particle correlations, multi-particle cumulants [19] have also been extensively used in $\mathrm{A}+\mathrm{A}$ and $p+\mathrm{A}$ collisions [20-22] to measure the $v_{n}$. The $n$-particle cumulant suppresses non-flow correlations involving $n$ or fewer particles, and is very effective in involving non-flow azimuthal correlations in $\mathrm{A}+\mathrm{A}$ and $p+\mathrm{A}$ collisions, as the non-flow correlations (such as those arising from jets, decays etc.) that only correlate few-particles among themselves. Thus it is natural to use the multiparticle cumulants in $p p$ collisions to study azimuthal anisotropies. However, the multiplicities in $p p$ collisions are so small that despite the suppression of such few particle correlations, they still affect the measured $v_{n}$. This inadequacy of the cumulants is measuring the $v_{n}$ in $p p$ collisions was recently demonstrated in Ref. [23], where the authors showed that the cumulants give significant and large non-zero values when analyzing MC events generated with the PYTHIA-8 [24] event generator. The authors of Ref. [23] proposed improved cumulant methods, called two-subevent and three-subevent cumulants, where the particles used in the cumulants are taken from two and three different $\eta$ regions, respectively. This $\eta$ separation, further suppresses jet-like correlations. Recently the ATLAS collaboration performed such subevent cumulant measurements in $p p$ collisions [25]. Figure 8 shows the multiplicity dependence of the three-subevent $v_{2}\{4\}$ measured in $5.02 \mathrm{TeV}$ and $13 \mathrm{TeV} p p$ collisions (left and middle panels) and compares them to the $v_{2}\{4\}$ seen in $5.02 \mathrm{TeV} p+\mathrm{Pb}$ collisions (right panel). Also shown for comparison are the $v_{2}$ values obtained from the $2 \mathrm{PCs}$. It is seen that the ordering $v_{2}-2 \mathrm{PC}>v_{2}\{4\}$ is observed in $p p$ collisions just as it was observed in the $p+\mathrm{Pb}$ and $\mathrm{Pb}+\mathrm{Pb}$ (Figure 3) collisions, again giving indications of collectivity in $p p$ collisions.

\section{Summary}

The measurement of long-range correlations in $p / \mathrm{D} / \mathrm{He}+\mathrm{A}$ collisions and their similarity to $\mathrm{A}+\mathrm{A}$ collisions is quite striking has established the presence of collective phenomena in such systems. Analysis of collectivity via measurement of the long-range correlation in $p p$ collisions is considerably more 


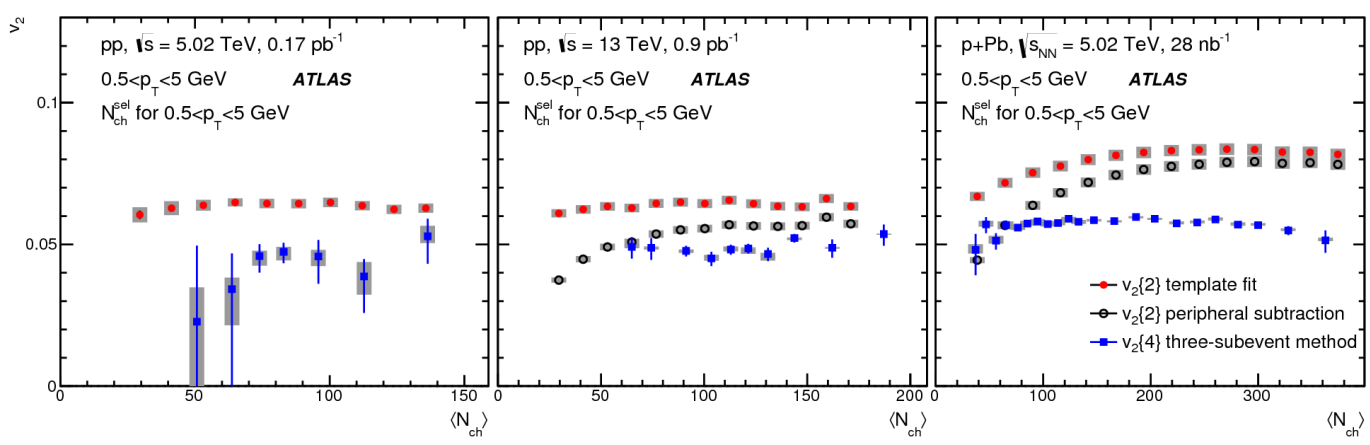

Figure 8. The $v_{2}\{4\}$ values calculated for charged particles with $0.5<p_{\mathrm{T}}<5 \mathrm{GeV}$ using the three-subevent method in $5.02 \mathrm{TeV} p p$ (left panel), $13 \mathrm{TeV} p p$ (middle panel) and $5.02 \mathrm{TeV} p+\mathrm{Pb}$ collisions (right panel). They are compared to the $v_{2}$ obtained from the $2 \mathrm{PC}$ analyses where the non-flow effects are removed by a template fit procedure (solid circles). Figure adapted from Ref. [25].

challenging. As described above, the most critical step in analyzing the long-range correlations in $p p$ collisions is the removal of correlations arising from jets and dijets. The standard cumulants that work quite well in A+A collisions fail in $p p$ collisions, as demonstrated in Ref. [25]. The subevent cumulants and template fitting procedure provide two effective methods of removing the jet and dijet correlations. However, even in these two methods, there might still be residual correlations from jets and dijets left over, so the results still must be taken with a pinch of salt. From the measurement, there are strong indications that global correlations - those that involve most of the particles produced in an event - are present in $p p$ collisions. These correlations quantified by the Fourier coefficients $v_{n}$, demonstrate no dependence on the collision energy over $\sqrt{s}$ of $2.76-13 \mathrm{TeV}$, similar to the $\sqrt{s_{\mathrm{NN}}}$ independence of the $v_{n}$ seen in A+A collisions. The correlations show no dependence on the multiplicity of the $p p$ collision either, i.e. they are just as strong in low-multiplicity events as they are in high multiplicity events. The subevent cumulant measurements show that the $v_{2}\{2\}$ is larger than $v_{2}\{4\}$, similar to that seen in A+A collisions, indicating the presence of event-by-event $v_{2}$ fluctuations. Further more, the $p_{\mathrm{T}}$ dependence of the $v_{2}$ seen in $p p$ collisions is qualitatively similar to that seen in $p+\mathrm{A}$ and $\mathrm{A}+\mathrm{A}$ collisions, in fact up to an overall scaling the $v_{2}\left(p_{\mathrm{T}}\right)$ look identical between $p p$, $p+\mathrm{Pb}$ and $\mathrm{A}+\mathrm{A}$ collisions. All these observations give strong indications of collective behavior in $p p$ collisions.

\section{Acknowledgments}

This work was supported by the US Department of Energy Office of Science, Office of Nuclear Physics under Award No. DE-FG02-86ER40281.

\section{References}

[1] ATLAS Collaboration, Phys. Rev. C 86, 014907 (2012)

[2] CMS Collaboration, JHEP 1009, 091 (2010)

[3] A. Dumitru, K. Dusling, F. Gelis, J. Jalilian-Marian, T. Lappi, R. Venugopalan, Phys. Lett. B 697, $21(2011)$ 
[4] ATLAS Collaboration, ATLAS-CONF-2016-105 (2016)

[5] ATLAS Collaboration, Phys. Rev. C96, 024908 (2017), 1609.06213

[6] ALICE Collaboration, B. Abelev et al., Phys. Lett. B 719, 29 (2013)

[7] ATLAS Collaboration, Phys. Rev. Lett. 110, 182302 (2013)

[8] CMS Collaboration, Phys. Lett. B 724, 213 (2013)

[9] ATLAS Collaboration, Phys. Rev. C 90, 044906 (2014)

[10] CMS Collaboration, Phys. Rev. Lett. 115, 012301 (2015)

[11] G. Başar, D. Teaney, Phys. Rev. C 90, 054903 (2014)

[12] PHENIX Collaboration, A. Adare et al., Phys. Rev. Lett. 111, 212301 (2013)

[13] PHENIX Collaboration, A. Adare et al., Phys. Rev. Lett. 114, 192301 (2015)

[14] STAR Collaboration, L. Adamczyk et al., Phys. Lett. B 747, 265 (2015)

[15] PHENIX Collaboration, A. Adare et al., Phys. Rev. Lett. 115, 142301 (2015)

[16] PHENIX Collaboration, C. Aidala et al. , Phys. Rev. C95, 034910 (2017)

[17] ATLAS Collaboration, Phys. Rev. Lett. 116, 172301 (2016)

[18] ALICE Collaboration, A. Jaroslav et al., Phys. Lett. B762, 376 (2016)

[19] N. Borghini, P.M. Dinh, J.Y. Ollitrault, Phys. Rev. C63, 054906 (2001)

[20] ATLAS Collaboration, Eur. Phys. J. C 74, 3157 (2014)

[21] ATLAS Collaboration, Phys. Lett. B 725, 60 (2013)

[22] ALICE Collaboration, Phys. Rev. Lett. 105, 252302 (2010)

[23] J. Jia, M. Zhou, A. Trzupek, Phys. Rev. C96, 034906 (2017)

[24] T. Sjöstrand, S. Mrenna, P.Z. Skands, Comput. Phys. Commun. 178, 852 (2008)

[25] ATLAS Collaboration (2017), arXiv 1708.03559 